\title{
Pediatric Brain Tumor Consortium
}

National Cancer Institute

\section{Source}

National Cancer Institute. Pediatric Brain Tumor Consortium. NCI Thesaurus. Code C19959.

Network of medical centers that will evaluate promising treatments for children with brain malignancies. 